\title{
Disciplina: História da Ciência - Tecnologia Nuclear, contribuição para a formação dos estudantes de pós-graduação em Engenharia
}

\section{Subject: Science History - Nuclear Technology, contribution to the education of graduate students in Engineering}

\section{Disciplina: Historia de la Ciencia - Tecnología Nuclear, contribución a la formación de los estudiantes de posgrado en Ingeniería}

http://dx.doi.org/10.221713/2358-2332.2016.v14.13721

Amir Zacarias Mesquita, doutor em Engenharia Química pela Universidade Estadual de Campinas (Unicamp), pesquisador titular do Centro de Desenvolvimento da Tecnologia Nuclear/Comissão Nacional de Energia Nuclear (CDTN/CNEN), docente permanente e membro do colegiado do Programa de Pós-Graduação em Ciência e Tecnologia das Radiações, Minerais e Materiais do CDTN, Belo Horizonte, MG, Brasil. E-mail: amir@cdtn.br.

Rubens Martins Moreira, doutor em Engenharia Química pela North Carolina State University, Estados Unidos, pesquisador titular do Centro de Desenvolvimento da Tecnologia Nuclear/Comissão Nacional de Energia Nuclear (CDTN/CNEN), docente permanente do Programa de Pós-Graduação em Ciência e Tecnologia das Radiações, Minerais e Materiais do CDTN, Belo Horizonte, MG, Brasil. E-mail: rubens@cdtn.br.

\section{Resumo}

Este artigo apresenta as atividades e inovações efetivadas com a inserção na grade curricular de um programa de pós-graduação da área de Engenharia II, na classificação da Capes, de uma disciplina de ciências humanas. A disciplina foi denominada "História da Ciência - Tecnologia Nuclear”, e o programa é o de Pós-Graduação em Ciência e Tecnologia das Radiações, Minerais e Materiais, do Centro de Desenvolvimento da Tecnologia Nuclear (PPG-CDTN). O CDTN é uma instituição de pesquisa da Comissão Nacional de Energia Nuclear e localiza-se em Belo Horizonte. O curso possui mestrado e doutorado e abrange as áreas de Ciência e Engenharia de Materiais; Física e Química da Matéria Condensada; Física e Biologia na Saúde; Geociências e Tecnologia Mineral; Aplicações de Técnicas Nucleares e Reatores Nucleares. Com essa disciplina, busca-se apresentar ao aluno um enfoque mais humanístico, político e social da evolução da ciência no campo da tecnologia nuclear. Pretendese, assim, integrar, à formação técnica do aluno de pós-graduação, uma visão crítica com valores e princípios, possivelmente ausente em sua graduação.

\footnotetext{
${ }^{1}$ Como citar: ABNT NBR 6023:2002 e incluir o DOI.
} 
Mesquita e Moreira / Disciplina: história da Ciência - Tecnologia Nuclear, contribuição para a formação dos estudantes de pós-graduação em Engenharia

Palavras-chave: Pós-Graduação. Ensino. Engenharia. História da Ciência. Tecnologia Nuclear.

\begin{abstract}
This article presents the activities and innovations performed by the inclusion of a Human Sciences subject in the curriculum of a graduate program in the field of Engineering II, according to the Capes classification. The subject was titled "Science History - Nuclear Technology", and the program is the Graduate Program in Science and Technology of Radiation, Minerals and Materials of the Nuclear Technology Development Center (PPGCDTN). CDTN is a research institution of the Brazilian Nuclear Energy Commission and is located in Belo Horizonte, in the state of Minas Gerais. The program has master's and doctorate degrees and comprises the areas of Materials Science and Engineering; Physics and Chemistry of Condensed Matter; Physics and Biology in Health; Geosciences and Mineral Technology; and Applications of Nuclear Techniques and Reactors. With this subject, the student is presented with a more humanistic, political and social approach to the evolution of science in the field of nuclear technology. It is intended, therefore, to integrate into the technical training of graduate students a critical vision with values and principles that, possibly, they did not have in their undergraduate education.
\end{abstract}

Keywords: Graduate Program. Teaching. Engineering. Science History. Nuclear Technology.

\title{
Resumen
}

Este texto presenta las actividades e innovaciones realizadas con la inclusión en el plan de estudios de posgrado en Ingeniería II, en la clasificación Capes, de una disciplina de humanidades. La disciplina se llama Historia de la Ciencia-Tecnología Nuclear, y el programa es el Posgrado en Ciencia y Tecnología de la Radiación, Minerales y Materiales, del Centro de Desarrollo de Tecnología Nuclear (PPG-CDTN). El CDTN es una institución de investigación de la Comisión Brasileña de Energía Nuclear y se encuentra en Belo Horizonte. El curso tiene maestría y doctorado y cubre las áreas de la Ciencia e Ingeniería de Materiales; Física y Química de la Materia Condensada; Física y Biología en Salud; Ciencias de la Tierra y la Tecnología Mineral, Aplicaciones de Ingeniería Nuclear y Reactores Nucleares. Por intermedio de esta disciplina se pretende proporcionar a los estudiantes un enfoque más humanista, político y social de la evolución de la ciencia, especialmente en el campo de la tecnología nuclear. Se pretende, por lo tanto, agregar a la formación técnica de los estudiantes de posgrado una visión crítica con los valores y principios que, posiblemente, no les habían presentado en la graduación.

Palabras clave: Posgrado. Enseñanza. Ingeniería. Historia de la Ciencia. Tecnología Nuclear. 
Mesquita e Moreira / Disciplina: história da Ciência - Tecnologia Nuclear, contribuição para a formação dos estudantes de pós-graduação em Engenharia

\section{INTRODUÇÃO}

O Programa de Pós-Graduação em Ciência e Tecnologia das Radiações, Minerais e Materiais (PPG-CDTN) é um curso, na classificação da Capes, da subárea de Engenharia Nuclear, área de Engenharia II, da grande área das Engenharias. O programa iniciou suas atividades com mestrado acadêmico, em fevereiro de 2003, no Centro de Desenvolvimento da Tecnologia Nuclear (CDTN), em Belo Horizonte. O CDTN é uma das unidades de pesquisa da Comissão Nacional de Energia Nuclear (CNEN), autarquia vinculada ao Ministério da Ciência, Tecnologia, Inovações e Comunicações. Em agosto de 2010, iniciou-se o curso de doutorado no programa. O PPG-CDTN possui quatro áreas de concentração: Ciência e Tecnologia de Materiais; Ciência e Tecnologia das Radiações; Ciência e Tecnologia dos Minerais e Meio Ambiente; e, por fim, Ciência e Tecnologia de Reatores Nucleares.

O CDTN localiza-se no campus universitário da Universidade Federal de Minas Gerais (UFMG), no bairro Pampulha, em Belo Horizonte, atuando em pesquisa e desenvolvimento, ensino e prestação de serviços (na área nuclear e em áreas correlatas). As principais atividades do Centro envolvem atualmente as áreas de tecnologia nuclear, minerais e materiais, saúde e meio ambiente. Nas aplicações das radiações e técnicas nucleares, destacam-se: o tratamento de rejeitos radioativos; a monitoração e remediação ambiental; a metrologia das radiações; o desenvolvimento e produção de radiofármacos para aplicações em tomografia por emissão de pósitrons; o aperfeiçoamento de processos de extração e purificação mineral; a nanotecnologia; a integridade estrutural e os serviços em radiologia. Há forte cooperação com os setores de energia, saúde, indústria do petróleo e meio ambiente (CENTRO DE DESENVOLVIMENTO DA TECNOLOGIA NUCLEAR, 2017).

O Centro é considerado uma instituição de pesquisa de grande porte e ocupa uma área de 240 mil metros quadrados, dos quais 42 mil são de área construída. Ele possui um reator nuclear de pesquisa do tipo TRIGA (Training, Research, Isotopes, General Atomics), uma unidade de pesquisa e produção de radiofármacos, um laboratório de irradiação gama, instalações-piloto para processamento de bens minerais, além de um parque laboratorial com cerca de 50 laboratórios de ensaios físicos e químicos (CENTRO DE DESENVOLVIMENTO DA TECNOLOGIA NUCLEAR, 2016).

O CDTN tem forte atuação na formação de recursos humanos em áreas estratégicas, por meio do Programa de Pós-Graduação em Ciências e Tecnologia das Radiações, Minerais e Materiais, nas modalidades de mestrado e doutorado acadêmicos. Há também um amplo programa de bolsas de iniciação científica (graduação) e de cursos de curta duração nas suas áreas de competência. O CDTN sempre atuou em treinamentos especializados na área nuclear, como em cursos de proteção radiológica para profissionais da saúde, das forças armadas, da docência etc. Ministrado no Centro desde 1974, o Curso de Treinamento de Operadores de Reatores de Pesquisa (CTORP) se destaca. Por intermédio dessa capacitação, cerca de 250 operadores e gerentes das centrais nucleares brasileiras Angra $1 \mathrm{e} 2$ foram treinados. O CTORP é um curso de um mês, prático, em que o aluno tem oportunidade de operar o reator de pesquisa TRIGA do CDTN. 
Mesquita e Moreira / Disciplina: história da Ciência - Tecnologia Nuclear, contribuição para a formação dos estudantes de pós-graduação em Engenharia

A atuação do reator IPR-R1 foi muito importante para o programa nuclear brasileiro na década de 1970, quando se realizou a caracterização do minério de urânio da primeira jazida brasileira, em Poços de Caldas (MG) (MESQUITA et al., 2011).

O PPG-CDTN é um curso de mestrado e doutorado acadêmicos. O objetivo é formar profissionais com alta qualificação científica, preparados tecnologicamente por meio de atividades didáticas avançadas e de pesquisa para o exercício de atividades profissionais. Os pós-graduandos são habilitados para atuar em atividades de pesquisa, desenvolvimento e inovação, tanto do setor nuclear como em áreas de pesquisa não convencionais de relevância estratégica para o estado de Minas Gerais e para o Brasil. Os futuros mestres e doutores são capacitados para contribuir no desenvolvimento de processos, na utilização eficiente de tecnologias avançadas e na formação de novos profissionais, tanto no setor produtivo especializado como em instituições de ensino e pesquisa (CENTRO DE DESENVOLVIMENTO DA TECNOLOGIA NUCLEAR, 2017).

O programa oferece os cursos de mestrado e de doutorado, os quais têm por finalidade, no mestrado, aprofundar as competências adquiridas nos cursos de graduação, aumentando o conhecimento e desenvolvendo o domínio de técnicas de investigação aplicáveis às respectivas áreas de concentração; e, no doutorado, proporcionar formação científica e tecnológica ampla e aprofunda, consolidando a autonomia para a pesquisa e o desenvolvimento tecnológico, bem como a capacidade crítica nas respectivas áreas de concentração (CENTRO DE DESENVOLVIMENTO DA TECNOLOGIA NUCLEAR, 2017).

$O$ enfoque de formação do PPG-CDTN se fundamenta em um programa multidisciplinar, apesar de estar classificado na Capes na área de Engenharia II e subárea de Engenharia Nuclear. Para se inscrever no exame de seleção, o candidato a discente deve ser graduado em uma das seguintes áreas: Engenharias, Ciências Exatas e da Terra, Ciências Biológicas, Ciências da Saúde ou Ciências Agrárias. Deste modo, o curso abrange: as áreas de Ciência e Engenharia de Materiais; Reatores Nucleares; Física e Química Experimental da Matéria Condensada; Física e Biologia na Saúde; Geociências e Tecnologia Mineral; e, por fim, Aplicações de Técnicas Nucleares em Meio Ambiente, Indústria e Medicina. Estas são áreas que empregam radiações e possuem profundo entendimento das interações entre radiação e matéria. Em 2012, foi criada uma nova área de concentração em tecnologia de reatores nucleares, abrangendo o estudo e a utilização da energia liberada pelo núcleo atômico.

A história da tecnologia nuclear se confunde com a própria história da humanidade. Desde os tempos antigos, o homem busca respostas para a pergunta: "Do que são feitas as coisas?" Esta questão mobilizou durante séculos o pensamento dos filósofos. Há três mil anos, os gregos começaram a refletir sobre o tema, mas, a partir do final dos anos 1800 e início dos anos 1900, uma sucessão de descobertas iria mudar a ciência radicalmente. A gênese da tecnologia nuclear é um dos capítulos mais fascinantes da história da ciência e tecnologia. Começou com Röntgen, com os raios-x (1895), Becquerel e a família Curie, com a radiação natural (1896), chegando, finalmente, à descoberta e utilização da maior força da natureza a energia do núcleo. Uma vez descoberta a radioatividade, o progresso científico foi extraordinariamente rápido, muito mais intenso que em qualquer período anterior da história da ciência. Becquerel, diga-se de passagem, foi contemporâneo de Joule e Kelvin na Física e de Darwin na Biologia. 
Mesquita e Moreira / Disciplina: história da Ciência - Tecnologia Nuclear, contribuição para a formação dos estudantes de pós-graduação em Engenharia

Hoje o homem domina e controla o processo da fissão nuclear. Segundo a Agência Internacional de Energia Atômica (International Atomic Energy Agency - IAEA) (2015), as usinas nucleares fornecem $16 \%$ da eletricidade mundial. Usinas nucleares se tornaram realidade em mais de 30 países, incluindo o Brasil, e é a única fonte capaz de suprir, por centenas de anos, as necessidades energéticas mundiais, sem emissão de gases que provocam o efeito estufa. São cerca de 440 usinas nucleares gerando eletricidade e mais de 240 reatores de pesquisa em operação. Ironicamente, Rutherford, o genial físico das primeiras reações nucleares, não acreditava que a energia nuclear pudesse algum dia ser usada. "Os gênios, afinal, também se enganam, incluindo sobre o futuro do assunto em que se tornaram geniais" (FIOLHAIS, 2016).

Atualmente, 16 países dependem da energia nuclear para produzir mais de um quarto de suas necessidades de eletricidade. Encontram-se em construção no mundo 53 usinas nucleares (Angra 3 é uma delas) e outras 135 foram encomendadas. Além delas, cerca de 300 estão sendo consideradas até 2030 pelo planejamento energético de vários países, incluindo o Brasil, que pretende construir oito usinas neste período.

São conhecidos mais de três mil isótopos ${ }^{2}$ dos 92 elementos químicos mais comuns da natureza. A maior parte dos isótopos é produzida nos aceleradores de partículas e nos reatores nucleares, cerca de 300 destes existem na natureza. A atual medicina seria inconcebível sem o uso de mais de uma centena destes isótopos (neste caso radioisótopos). Aproximadamente 10 mil hospitais em todo o mundo usam radioisótopos para realizar mais de 30 milhões de procedimentos médicos por ano. São utilizados no tratamento do câncer, nas tomografias, nos exames de ressonância magnética e em outras técnicas de diagnóstico por imagem. As aplicações nucleares pacíficas incluem erradicação de insetos causadores de doenças e a produção de cultivares induzidos pela radiação que melhoraram o rendimento das culturas alimentares no mundo em desenvolvimento.

Nos dias atuais, o estudo do comportamento do núcleo e a análise das forças nucleares prosseguem, já que são ainda inúmeros os problemas em aberto. Experimentos são realizados para sintetizar novos elementos situados acima do urânio na tabela periódica (transurânicos), para tentar isolar os quarks ${ }^{3}$ e para reproduzir de modo controlado (e rentável) a produção energética das estrelas (fusão nuclear).

Este artigo descreve as atividades e as inovações efetivadas com a inserção da disciplina História da Ciência - Tecnologia Nuclear, da área de conhecimento em Ciências Humanas, na grade curricular do Programa de Pós-Graduação em Ciência e Tecnologia das Radiações, Minerais e Materiais do Centro de Desenvolvimento da Tecnologia Nuclear (PPG-CDTN), da área de Engenharias II (Capes).

\section{BREVE HISTÓRIA DO CDTN}

As atividades pioneiras em tecnologia nuclear no Brasil iniciaram-se em 1952 (Governo Vargas), no Instituto de Pesquisas Radioativas (IPR), uma unidade da Escola de Engenharia

\footnotetext{
${ }^{2}$ Isótopos são átomos de um mesmo elemento químico (mesma quantidade de prótons), mas com número de nêutrons diferentes.

${ }^{3}$ Quarks são partículas subatômicas que se combinam em número de três para formar os prótons e os nêutrons.
} 
Mesquita e Moreira / Disciplina: história da Ciência - Tecnologia Nuclear, contribuição para a formação dos estudantes de pós-graduação em Engenharia

(campus da UFMG, Pampulha), da então Universidade de Minas Gerais, hoje UFMG. Sua criação foi liderada pelo engenheiro Francisco de Assis Magalhães Gomes, seu primeiro diretor. Francisco Magalhães, além de engenheiro, foi também físico, educador, político, humanista e historiador. As atividades iniciais do IPR incluíam a pesquisa de ocorrências de minerais radioativos e estudos em Física e Química Nuclear, metalurgia e materiais de interesse nuclear. Em 1960, entrou em operação o reator nuclear de pesquisa TRIGA IPR-R1, mostrado na Figura 1. O reator TRIGA foi adquirido pelo governo do estado de Minas Gerais por meio do programa do governo americano Átomos para a Paz. Foi o segundo reator nuclear instalado no país.

\section{Figura 1 - Reator nuclear de pesquisa TRIGA IPR-R1 do CDTN}
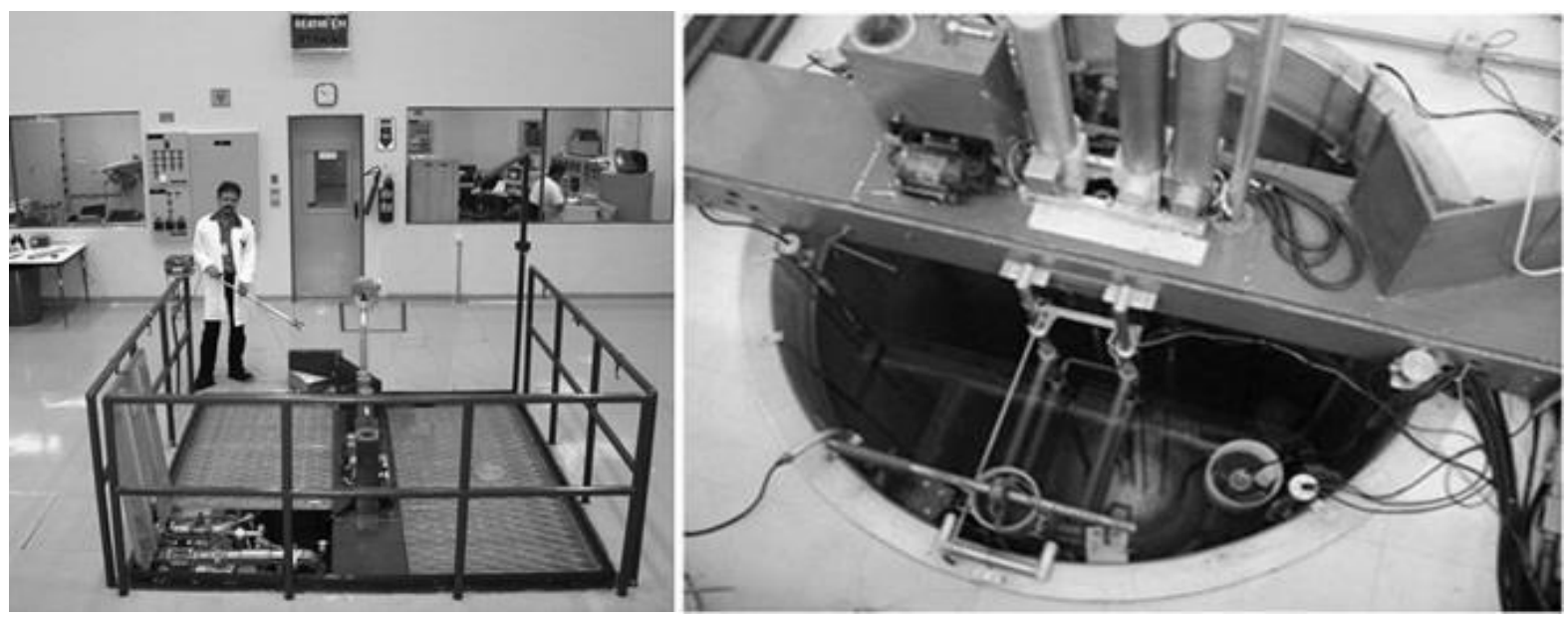

Fonte: Mesquita et al. (2011).

Nota: a)primeira figura da esquerda para direita: Operador do reator retirando amostra irradiada. b) segunda figura da esquerda para direita: Reator em operação, vendo no fundo da piscina a radiação de Cerenkov.

Átomos para a Paz foi o título do discurso do presidente Eisenhower na Assembleia Geral das Nações Unidas em 1953. Nesse mesmo ano ocorreu a morte do ditador soviético Stalin e a Guerra Fria estava em seu auge. O discurso foi um ponto de inflexão para o foco internacional sobre os usos pacíficos da energia atômica, durante as fases iniciais da Guerra Fria. O presidente Eisenhower se dirigiu, especificamente, para os países em desenvolvimento. A energia nuclear foi apresentada como um meio para alcançar maiores níveis de progresso e bem-estar em todo o mundo. O programa forneceu a base ideológica para a criação da IAEA e para o Tratado de Não Proliferação de Armas Nucleares (RÖHRLICH, 2013).

No final de 1965, iniciaram-se os trabalhos do Grupo do Tório no IPR, dentro da Divisão de Engenharia de Reatores deste instituto. A principal atribuição deste grupo era estudar as possibilidades técnicas e econômicas da utilização do tório em um programa nuclear de longo prazo. Nesta mesma época, a CNEN iniciou o debate sobre qual seria a linha de reatores que o Brasil deveria utilizar. Coube ao Instituto de Engenharia Nuclear, no estado do Rio de Janeiro, analisar o reator de alta temperatura. O Instituto de Energia Atômica - hoje Instituto de Pesquisas Energéticas e Nucleares - e o IPR ficaram com a tarefa de estudar o reator de água pesada ${ }^{4}$ e urânio natural. Esta foi uma grande controvérsia da área nuclear nessa

${ }^{4}$ Água pesada $\left(\mathrm{D}_{2} \mathrm{O}\right)$ : água que contém uma proporção significativamente maior de átomos de hidrogênio pesad 
Mesquita e Moreira / Disciplina: história da Ciência - Tecnologia Nuclear, contribuição para a formação dos estudantes de pós-graduação em Engenharia

época, pela possibilidade de se fazer artefatos bélicos. O reator que utiliza como combustível o urânio natural, mediante ativação neutrônica do urânio-238, forma o plutônio-239. Era época do Governo Militar no Brasil e este tema era bastante polêmico (CAMARGO, 2006).

Para dar partida a um reator tendo como combustível o tório (material fértil), é preciso de uma adequada quantidade de material físsil, ou seja, urânio-235 (urânio enriquecido) ou plutônio-239 (criado pela captura de um nêutron pelo isótopo urânio-238). Não existiam, na época, instalações de enriquecimento de urânio no Brasil. Assim para projetar um reator a tório, era necessário projetar e montar um reator a urânio natural e água pesada ${ }^{5}$. Para conseguir o isótopo físsil (Pu-239) para a partida do reator a tório, construiu-se um reator de pesquisa a água pesada, o Capitu (Circuito de Água-Pesada Tório-Urânio). O objetivo final era a construção de um reator regenerador rápido ${ }^{6}$ (fast breeder), que transforma tório-232 (isótopo fértil) em urânio-233 (isótopo físsil). Este isótopo do urânio praticamente não existe na natureza.

Segundo Camargo (2006), a importância do uso do tório como combustível se devia aos seguintes aspectos: o minério ter reservas maiores do que o urânio; o elevado consumo de urânio pelos reatores normais esgotaria as reservas e o preço do urânio iria subir rapidamente; além de o Brasil possuir as maiores fontes de tório do planeta e, portanto poderia se tornar líder mundial nesta linha de reatores.

O Grupo do Tório se destacou pela formação de mestres e doutores nos países desenvolvidos e pelas publicações técnicas relevantes na área de reatores nucleares. $\mathrm{O}$ grupo fazia parte de um programa de cooperação com a França. Pesquisadores foram enviados para treinamento nesse país na área de transferência de calor em reatores. O Brasil nunca havia projetado um reator nuclear de potência. O Grupo do Tório era formado por engenheiros que queriam aproveitar a abundância desse elemento no país ${ }^{7}$. A comunidade de pesquisadores nucleares do Brasil era formada por investigadores que atuavam com Física Teórica e Física Aplicada. O diferencial do Grupo do Tório foi a formação de um núcleo de engenheiros entre o grande número de físicos da época. Os engenheiros davam importância à transferência de calor, ao fluxo de nêutrons, à metalurgia, aos materiais estruturais etc.

Assim, foram originadas as primeiras instalações nacionais de pesquisa relativas aos reatores de potência. Foram montados, no IPR (hoje CDTN), um laboratório de termohidráulica, uma unidade subcrítica e um laboratório de testes de componentes nucleares. Foram compradas seis toneladas de água pesada nos Estados Unidos. A equipe realizou vários experimentos de física de reatores e construiu várias montagens experimentais de reticulados com água pesada nestes laboratórios, para posicionamento de combustíveis a urânio natural.

o (deutério - H com um nêutron), em relação aos átomos de hidrogênio (apenas com um próton). Água pesada é utilizada como moderador devido a sua eficácia na redução da velocidade dos nêutrons, possibilitando a reaç ão em cadeia em reatores com urânio natural (não enriquecido).

${ }^{5}$ O minério urânio é composto de 0,7\% de U-235 (material físsil) e 99,3\% de U-238 (material fértil). Utilizando água pesada consegue-se manter as reações em cadeia com o U-235 e o U-238 recebe um nêutron e se transforma em Pu-239, que é um material físsil. Enriquecimento do urânio é o aumento da porcentagem do isótopo U-235 no combustível.

${ }^{6}$ Os reatores regeneradores utilizam os nêutrons rápidos para realizarem as fissões, ao contrário dos reatores térmicos, que utilizam os nêutrons lentos ou termalizados para provocarem as fissões nucleares.

${ }^{7}$ As areias monazíticas das praias do estado do Espírito Santo, têm em sua composição elevada quantidade de tório, além de urânio. 
Mesquita e Moreira / Disciplina: história da Ciência - Tecnologia Nuclear, contribuição para a formação dos estudantes de pós-graduação em Engenharia

Houve grande quantidade de trabalhos publicados e comparados com resultados internacionais. O grupo alcançou grande notoriedade na época, principalmente entre os segmentos nacionalistas brasileiros (CAMARGO, 2006).

Mas o marco fundamental da história da indústria nuclear no Brasil foi a aquisição turnkey da Central Nucleoelétrica de Angra 1, comprada da americana Westinghouse Electric Corporation, em 1972. Essa decisão vinculou definitivamente o desenvolvimento da indústria nuclear brasileira à tecnologia dos reatores PWR (Pressurised Water Reactor), tipo de reator bem-sucedido no mundo desenvolvido e adotado por mais de $85 \%$ das usinas então existentes. A opção do governo brasileiro pelo reator a urânio enriquecido e água leve abortou as pesquisas em andamento pelo Grupo do Tório e este foi extinto. O grande salto no desenvolvimento da indústria nuclear no país se deu com o acordo nuclear Brasil-Alemanha, em 1975, no qual o Brasil previa a construção de oito centrais nucleares até o ano de 1990, bem como o domínio e industrialização de todo o ciclo do combustível nuclear - mineração e beneficiamento; enriquecimento; produção de pó e pastilhas de óxido de urânio; produção do elemento combustível; projeto, construção e operação de centrais nucleares; reprocessamento do combustível irradiado; tratamento dos rejeitos radioativos (FILGUEIRAS, 2002) (KAMIOJI, e MAGALHÃES, 2015).

O IPR foi separado da UFMG e transferido para a Companhia Brasileira de Tecnologia Nuclear (CBTN) em 1972. Em 1974, foi incorporado pela companhia estatal Empresas Nucleares Brasileiras S/A (Nuclebrás) e, em 1977, teve sua denominação alterada para Centro de Desenvolvimento da Tecnologia Nuclear (CDTN), com o papel de apoiar o desenvolvimento tecnológico das unidades industriais da Nuclebrás, absorvendo a tecnologia transferida no âmbito do acordo nuclear Brasil-Alemanha (1975-1988). Com a extinção da Nuclebrás, em 1988, o IPR voltou a fazer parte da CNEN e a ter uma atuação mais voltada para P\&D e para formação especializada na área nuclear e em áreas correlatas. Nesta nova fase, o CDTN estabeleceu maior cooperação com outras instituições de pesquisa, com a indústria e órgãos de governo, expandindo sua interação com a sociedade. Em 2003, o CDTN iniciou o programa de pós-graduação, em nível de mestrado, e, em 2010, o de doutorado, de significante relevância para a formação de novas gerações de pesquisadores (CENTRO DE DESENVOLVIMENTO DA TECNOLOGIA NUCLEAR, 2016).

\section{AS CIÊNCIAS HUMANAS EM CURSOS DE ENGENHARIA}

O engenheiro é um profissional preocupado com a aplicação do conhecimento científico e matemático e possui criatividade para desenvolver soluções de problemas técnicos e aplicá-los no mundo em que vivemos, a fim de suprir as necessidades e favorecer o desenvolvimento da sociedade. Engenheiros projetam materiais, estruturas e sistemas, considerando as limitações impostas pela praticidade, regulamentação, segurança e custo. Nos processos de criação, aperfeiçoamento e complementação, a engenharia conjuga os vários conhecimentos especializados no sentido de viabilizar as utilidades, tendo em conta a sociedade, a técnica, a economia e o meio ambiente. Independentemente da área de atuação, o engenheiro deve atuar com responsabilidade social (PEINADO; RODACOSKI, 2008).

Não só o engenheiro, mas também os profissionais de outras áreas do conhecimento (como Ciências Exatas e da Terra, Ciências Biológicas, Ciências da Saúde e Ciências Agrárias) 
Mesquita e Moreira / Disciplina: história da Ciência - Tecnologia Nuclear, contribuição para a formação dos estudantes de pós-graduação em Engenharia

devem desenvolver uma visão sistemática do mundo para reconhecerem-se como agentes de transformação social. Assim, não basta dominar os conhecimentos empíricos, tecnológicos e científicos, deve-se, adicionalmente, saber aplicar esses conhecimentos de forma ética e humana. Os profissionais dessas áreas devem estar comprometidos no desenvolvimento de suas tarefas com total responsabilidade social, seja qual for sua área de atuação. Particularmente, o engenheiro deverá ser capaz de criar mecanismos, produtos, processos e estruturas no intuito de transformar recursos naturais e não naturais para satisfazer as necessidades humanas. Um profissional deste ramo deve apresentar uma visão aberta, crítica, humanista, generalista e ecologicamente correta para poder absorver e desenvolver novas tecnologias com objetivo de solucionar os problemas em atendimento às demandas da sociedade (SILVA FILHO; SANTANA; SILVA, 2011).

A ciência está inserida no nosso dia a dia, quando nos comunicamos, quando estudarmos, quando escolhemos os alimentos e medicamentos para consumo. Portanto, no nosso cotidiano, não lembramos que para chegar até a atualidade foram anos de pesquisa por cientistas que, muitas vezes, não sabemos quais foram e qual contexto levou ao produto ou à técnica utilizada no momento. A partir do final do século XVIII, a ciência passou a fazer parte de todos os atos da vida humana. Na época do Iluminismo, a ciência ocupava papel de destaque. Era a partir de um rigoroso planejamento científico que os governantes acreditavam ser possível administrar seus reinos. Assim, dava-se muita importância ao estudo e, consequentemente, aconselhavam-no a todos que quisessem ocupar cargos diretivos. No século XIX, a concepção de ciência começa a se parecer com o que se tem hoje. Entende-se, porém, que tal concepção já estava elaborada desde muito tempo. No entanto, há autores que se referem ao período renascentista e outros que retrocedem à Idade Média para encontrar sua origem. Entre as características da ciência moderna estava a crença de que esta levaria à verdade e à certeza. Hoje em dia já não se compartilha mais de tal crença, mas a ciência ainda é fonte de segurança para o homem contemporâneo, de tal forma que ainda se procura nela, talvez não mais verdades ou certezas, mas uma opinião isenta e abalizada (ROSSONI, 2003) (PEINADO e RODACOSKI, 2008).

\section{A DISCIPLINA: HISTÓRA DA CIÊNCIA - TECNOLOGIA NUCLEAR}

A Filosofia, a Sociologia e a História são de grande relevância e devem, cada vez mais, serem oferecidas como disciplinas nos cursos de Engenharia, como elo entre as Ciências Exatas e as Humanas (SANTOS et al., 2014). O PPG-CDTN, conforme já mencionado, está incluído na área de Engenharia II na classificação da Capes, mas o programa abrange áreas distintas que vão desde Ciências Exatas até Ciências da Saúde, isto é, tenta englobar vários campos de aplicação das radiações ionizantes. Portanto, um programa de ensino multidisciplinar, como o do CDTN, para ser inovador, moderno e atual, não poderia prescindir dessa matéria.

Desse modo, os autores deste artigo propuseram ao colegiado do PPG-CDTN a inclusão da disciplina: História da Ciência - Tecnologia Nuclear, na grade curricular do programa. Inicialmente, houve discordância de alguns membros do colegiado. Alegava-se, entre outros motivos, que o enfoque não era compatível com o curso, que os discentes frequentariam a disciplina por ser simples e fácil e que eles optariam pela matéria em detrimento de outras 
Mesquita e Moreira / Disciplina: história da Ciência - Tecnologia Nuclear, contribuição para a formação dos estudantes de pós-graduação em Engenharia

disciplinas "mais importantes". Após as argumentações positivas de um dos autores deste artigo, que é membro do colegiado do PPG-CDTN, a disciplina foi aprovada em caráter experimental, mas com o valor de apenas um crédito, com uma carga horária total de 15 horas semestrais e como disciplina optativa. Em 2014, a disciplina foi ministrada no segundo semestre. Mesmo valendo apenas um crédito, ela foi oferecida com carga horária de 30 horas, devido ao interesse dos alunos e a abrangência dos temas apresentados. Foram cerca de 30 alunos nesse primeiro ano.

Em 2014, após alegações do professor responsável pela disciplina, o colegiado aprovou a aumento dos créditos para dois e a disciplina foi fornecida novamente, inclusive com um aluno de outro programa de pós-graduação. Finalmente, em 2016, o colegiado aumentou o valor dos créditos para quatro, com carga horária de 60 horas.

A disciplina está sendo ministrada em dois dias na semana por dois docentes permanentes do programa. Um deles possui graduação em Engenharia Elétrica, mestrado em Tecnologia Nuclear e doutorado em Engenharia Química. O outro docente possui graduação em Engenharia Química, mestrado em Tecnologia Nuclear e doutorado em Engenharia Química. Mesmo tendo os instrutores formação em Engenharia, estes profissionais, em suas trajetórias da vida, sempre tiveram grande interesse, atuação e sensibilidade por Ciências Humanas e Sociais.

Conforme relatado, o programa aceita graduados não só da área de Ciências Exatas e Engenharia, mas também graduados de área de Ciências Biológicas e Ciências da Saúde. Para estas duas áreas, o estudo de temas como a energia liberada pelo núcleo atômico, a equação de Schrödinger e a dualidade onda-partícula (De Broglie, Einstein) poderia parecer estranho à sua formação e seriam conteúdos de difícil assimilação por esses discentes. Mas, do modo que estes temas - importantes para a área nuclear - são desenvolvidos na disciplina, seu aprendizado tem sido agradável. Vários fatos interessantes são apresentados de modo aprazível, despertando a curiosidade dos discentes. Por exemplo, quando é apresentado o Princípio da Incerteza de Heisenberg ou "Princípio da Indeterminação". Deste princípio Einstein discordou pronunciando a sua famosa frase: "Deus não joga dados" (RONAN, 1987). Outro fato interessante exibido aos alunos refere-se a Werner Heisenberg, que foi o líder do programa de construção da bomba nuclear dos nazistas, motivando inclusive Niels Bohr a pôr fim na amizade entre eles. Assim, em paralelo ao ensino técnico, são apresentados os fatos relativos aos acontecimentos históricos, em uma sequência lógica, facilitando a assimilação dos dois enfoques.

Destaca-se que as aulas são ministradas em um moderno auditório, com recursos de multimídia). De modo geral, os professores fazem uma explanação inicial sobre o tema da aula, apresentam algum vídeo e, em seguida, ocorre um debate entre professor e alunos. Assim, pode-se avaliar o interesse dos alunos, fato que se tem mostrado bastante estimulador.

\footnotetext{
${ }^{8}$ Este princípio afirma que a suposição de que a energia se move em quanta discretos, significa que alguns pares de variáveis que, constantemente, afetam uma à outra, tais como tempo e energia, não podem ser determinados com precisão absoluta. Quanto mais precisamente uma é definida, menos precisamente se pode definir a outra.
} 
Mesquita e Moreira / Disciplina: história da Ciência - Tecnologia Nuclear, contribuição para a formação dos estudantes de pós-graduação em Engenharia

A disciplina História da Ciência - Tecnologia Nuclear foi dividida em duas partes. A primeira apresenta uma visão mais geral da jornada do homem na compreensão da natureza. Mostra o desenvolvimento da ciência nas diversas civilizações e culturas num cenário histórico. É dada uma visão do desenvolvimento do pensamento científico em todo o mundo, desde os tempos primitivos até a atualidade. Busca-se apresentar ao aluno um enfoque mais humanístico, político e social da evolução da ciência. Procura-se mostrar sua evolução, enfocando os fatos e personagens que levaram ao seu desenvolvimento.

A segunda parte destaca o grande impulso ocorrido no início do século XX na área da Tecnologia Atômica e Nuclear. São apresentados os grandes personagens, fatos, eventos, ideias e teorias no campo das ciências nucleares. Nas aulas são apresentados vídeos e realizados debates entre os alunos. Os temas apresentados se iniciam com as primeiras concepções do átomo pelos gregos até acontecimentos mais recentes, como os acidentes de Chernobyl e Fukushima, passando pelo Projeto Manhattan e os grandes personagens, como Mendeleev, os Curies, Fermi, Einstein etc. As aulas são ilustradas com documentários de veículos de comunicação, como Discovery Channel, National Geographic, History Channel, BBC etc. Como obras de referência recomendadas aos alunos, podem-se citar Ronan (1987), Tennenbaum (2007) e Camargo (2006).

Os tópicos da disciplina são dados de forma alternada nos dois dias da semana. Em um dia é apresentada a evolução de Ciência de modo mais amplo. No outro dia, o enfoque é a evolução da tecnologia nuclear. A seguir é apresentada a ementa atual da disciplina.

\section{Ementa $-1^{\mathrm{a}}$ Parte: $\underline{\text { História da Ciência }}$}

As origens: ciência primitiva.

Egito: Astronomia e Matemática.

Mesopotâmia: Escrita, Astronomia e Medicina.

Maias, Incas e Astecas: calendário.

Grécia: Homero, Hesíodo, Leucipo, Demócrito, Epicuro, Platão e Aristóteles.

Alexandria e a Ciência Helenística: Arquimedes.

Ciência chinesa.

Ciência hindu e indiana.

Idade de Ouro Islâmica (Ibn Sīnā “Avicena”).

Ciência Romana e Medieval.

Século XIX: Biologia, Química, Física, Matemática e Astronomia.

Século XX: Darwin, Mendel, bioquímica, biologia molecular.

\section{$\underline{\text { Ementa }-2^{\mathrm{a}} \text { Parte: Tecnologia Nuclear }}$}

Química, Tabela Periódica: Dalton e Mendeleev.

Mecânica Clássica: Galileu, Newton e Huygens.

Raios X, Radioatividade: Röntgen, Becquerel e os Curies.

Modelos Atômicos e Nucleares: Thomson, Rutherford e Bohr.

Teoria Quântica: Planck, Heisenberg, Dirac e Hawking.

Dualidade Onda-Partícula: de Broglie, Einstein e Schrödinger. 
Mesquita e Moreira / Disciplina: história da Ciência - Tecnologia Nuclear, contribuição para a formação dos estudantes de pós-graduação em Engenharia

Relatividade: Einstein, Eddington.

Nêutron: Chadwick.

Fissão Nuclear: Noddack, Hans, Strassmann, Meitner e Frisch.

Reação em Cadeia: Fermi, Compton.

Projeto Manhattan: Szilárd, Oppenheimer e Teller.

Acidentes Nucleares: Chernobyl e Fukushima.

Energia Nuclear no Brasil: Lattes.

As aulas não se limitam a apresentar apenas personagens e fatos históricos ligados à ciência, mas também conceitos técnicos. Deste modo, promove e facilita a compreensão das disciplinas obrigatórias que caracterizam o programa, como Física das Radiações, Proteção Radiológica, Geoquímica, Radioquímica, Radiologia, Reatores Nucleares, Radiobiologia etc., estando em concordância com o estudo:

\begin{abstract}
A história da ciência não pode substituir o ensino comum das ciências, mas pode complementá-lo de várias formas. O estudo adequado de alguns episódios históricos permite compreender as inter-relações entre ciência, tecnologia e sociedade, mostrando que a ciência não é uma coisa isolada de todas as outras, mas, sim, faz parte de um desenvolvimento histórico, de uma cultura, de um mundo humano, sofrendo e influenciando por sua vez muitos aspectos da sociedade. (MARTINS, 2006, p. 21)
\end{abstract}

Essa humanização quebra o paradigma de que, ainda segundo Martins (2006, p. 22), "[...] a ciência é algo atemporal, que surge de forma mágica e que está à parte de outras atividades humanas". Destaca-se aqui também a afirmação:

\begin{abstract}
Um dos possíveis caminhos para a melhoria do ensino das disciplinas científicas está na utilização da História e Filosofia da Ciência, podendo ser empregada como conteúdo em si, e/ou como estratégia para o ensino. Ainda salientamos que outras perspectivas relacionadas ao ensino apontam também para uma abordagem que trate dos conhecimentos histórico-filosóficos dos conteúdos das disciplinas científicas de uma forma que articulem a tríade Ciência, Tecnologia e Sociedade. (SANTOS et al., 2014, p. 76)
\end{abstract}

A Figura 2 apresenta um cartaz de divulgação da disciplina publicado nas redes sociais da internet. Os alunos aprendem as leis e teorias físico-químicas de modo lúdico, relacionandoas ao fato histórico que as envolve. 
Mesquita e Moreira / Disciplina: história da Ciência - Tecnologia Nuclear, contribuição para a formação dos estudantes de pós-graduação em Engenharia

\section{Figura 2 - Cartaz de divulgação da disciplina}

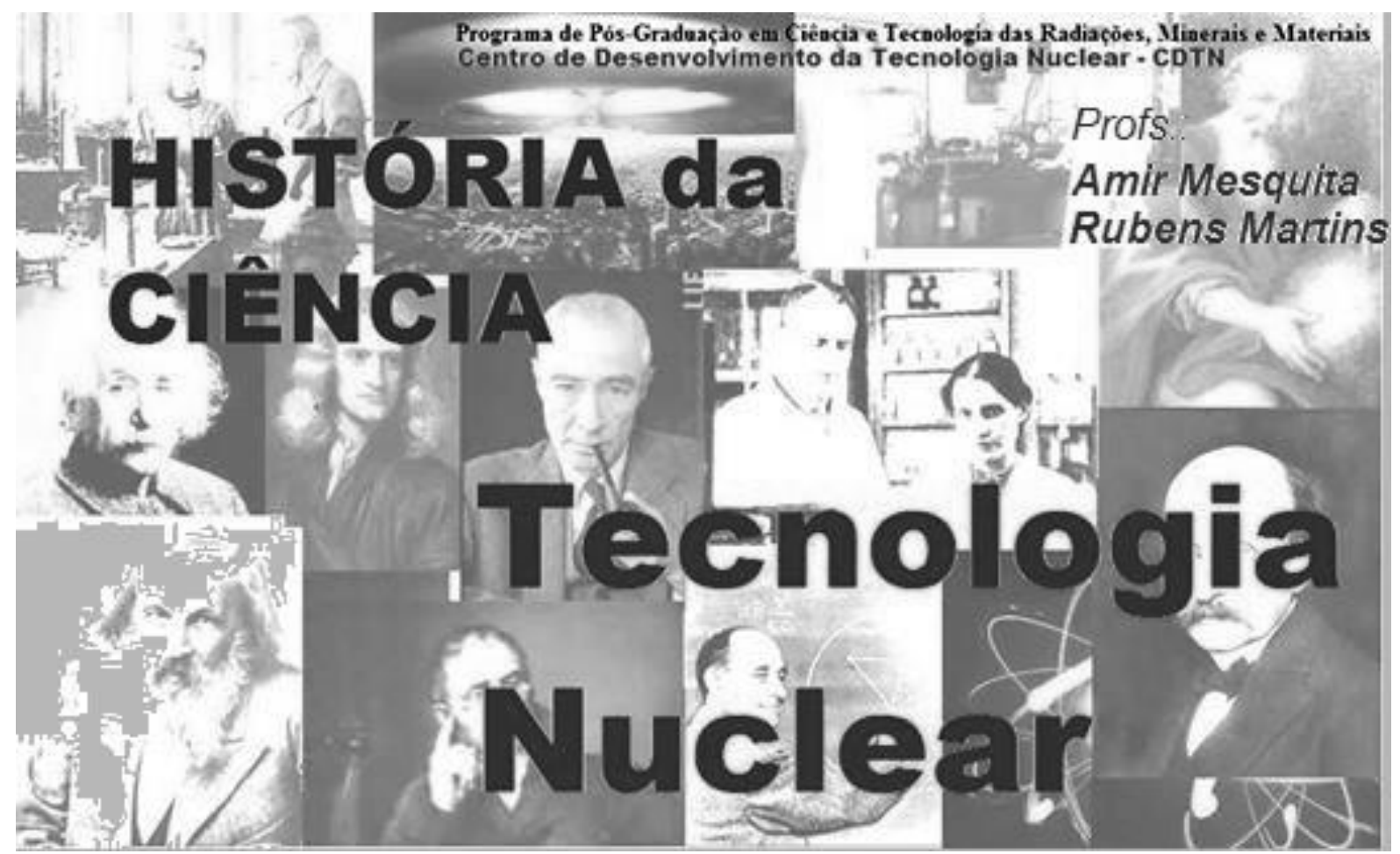

Fonte: Elaboração dos autores.

A Figura 3 mostra uma tela de um dos vídeos apresentados nas aulas. Esta imagem corresponde à série francesa "La Saga des Nobel” (LA TRIBU CURIE, 2014).

\section{Figura 3 - Recurso de mídia utilizado nas aulas}

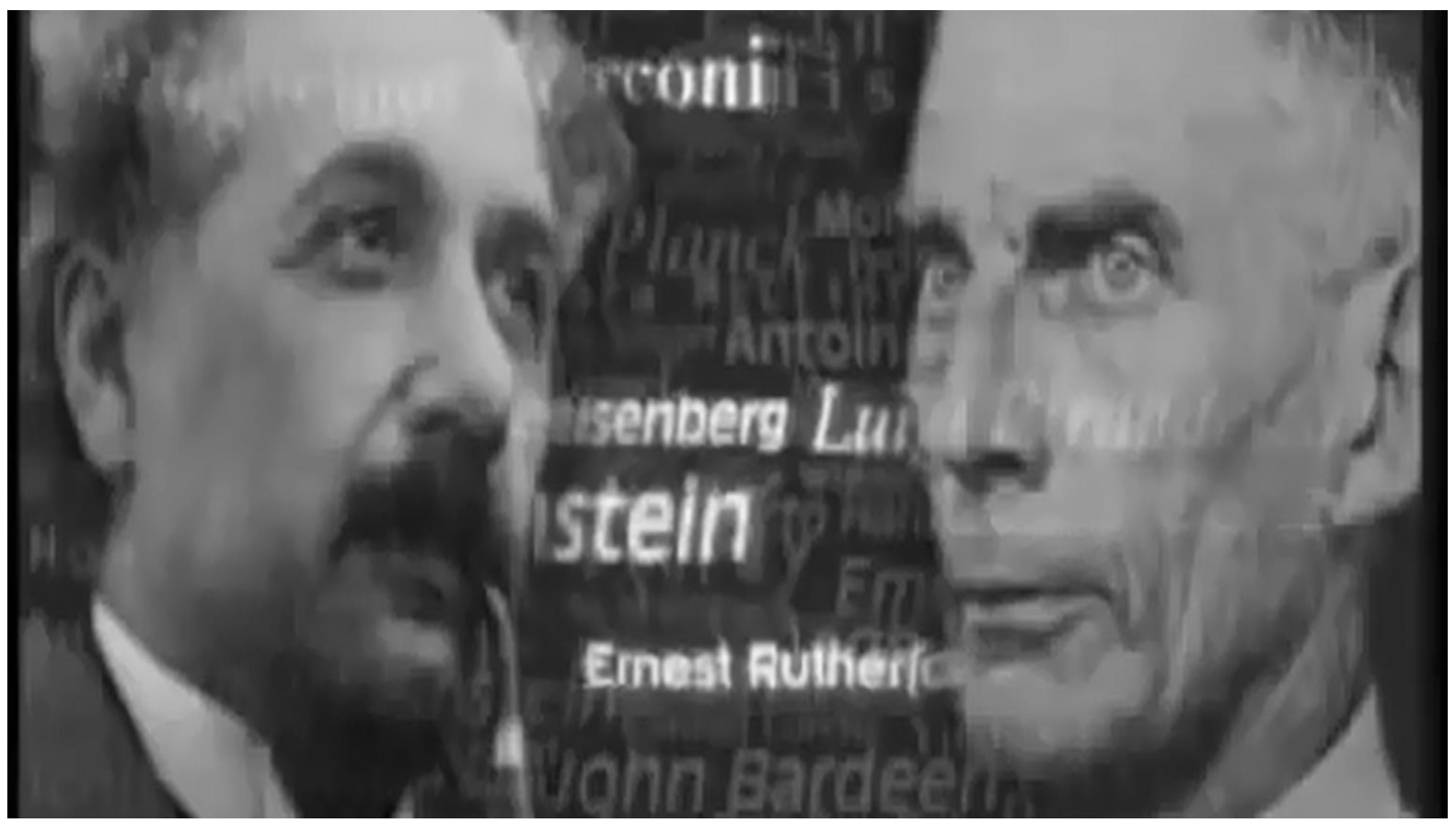

Fonte: La Tribu Curie (2014). 
Mesquita e Moreira / Disciplina: história da Ciência - Tecnologia Nuclear, contribuição para a formação dos estudantes de pós-graduação em Engenharia

\section{CONSIDERAÇÕES FINAIS}

A ciência é uma grande experiência intelectual. Atuar nela requer uma vívida imaginação criadora, temperada por firme disciplina, baseada num corpo consistente de observações comprovadas. A ciência atrai alguns dos melhores cérebros de cada civilização que se desenvolveu até o estágio em que lhe é possível enfrentar o desafio da natureza. A ciência não se resume apenas a coletar fatos, embora isto seja necessário. Ela é um sistema de correlação lógica dos fatos que, juntos, consolidam uma hipótese ou o corpo de uma teoria. A ciência é um conjunto de conhecimentos crescentes e em expansão, até o ponto em que aquelas mudanças são motivadas por experiências ainda mais complexas. Mas a história da ciência se prende a todas as oscilações da história mais geral (RONAN, 1987).

O Programa de Pós-Graduação em Ciência e Tecnologia das Radiações, Minerais e Materiais do CDTN/CNEN é um curso da área de Engenharia II na classificação da Capes. Nos últimos três anos, tem sido ministrada aos discentes a disciplina História da Ciência Tecnologia Nuclear. Tenta-se, assim, desenvolver nos futuros pós-graduados uma consciência mais humanista, social e crítica. Procura-se dar uma visão geral do desenvolvimento da ciência e do pensamento científico em todo o mundo, com destaque para o grande avanço que ocorreu quando o homem descobriu a energia e o potencial inovador que é o núcleo do átomo. A experiência da introdução desta disciplina tem sido um sucesso, com grande adesão e participação dos discentes.

O profissional de Engenharia e Ciências Exatas necessita ter formação de caráter técnico e humano e procurar integrá-las. Ao inserir disciplinas de ciências humanas na grade curricular de um curso de Engenharia, bem como relacionar o conteúdo técnico com suas aplicações e efeitos sociais e históricos, possibilita-se uma formação plena, completa, capaz de constituir um profissional socialmente responsável com maior capacidade de relacionamento interpessoal. Portanto, mostra-se necessária a reestruturação dos programas de Engenharia, tanto de graduação como de pós-graduação, com a inserção de disciplinas das Ciências Humanas na grade curricular. É necessário também mudança de postura dos docentes, garantindo uma visão mais questionadora e crítica do conteúdo transmitido. A formação do engenheiro, assim, transcenderá o ato de obter apenas o conhecimento restrito, puramente técnico, para possuir a sabedoria filosófica de mundo, de valores, de princípios e do próprio homem (SCRIGNOLI et al., 2008).

Uma das lições a serem aprendidas com a história das realizações científicas é que nem sempre as teorias sobrevivem para sempre. Muitas vezes, quando as coisas parecem solidificadas, novas observações e novas ideias são substituídas por conceitos atualizados. Isso faz parte da aventura que é a ciência, parte da lenta conquista do enigma que é o mundo natural. É a longa batalha pelo conhecimento, em que o homem está empenhado desde os primeiros dias da mais antiga civilização (RONAN, 1987). 
Mesquita e Moreira / Disciplina: história da Ciência - Tecnologia Nuclear, contribuição para a formação dos estudantes de pós-graduação em Engenharia

\section{Agradecimentos}

Ao Centro de Desenvolvimento da Tecnologia Nuclear (CDTN), à Comissão Nacional de Energia Nuclear (CNEN), à Fundação de Amparo à Pesquisa de Minas Gerais (Fapemig) e ao Conselho Nacional de Desenvolvimento Científico e Tecnológico (CNPq), pelo apoio prestado a esta pesquisa.

\section{Referências}

CAMARGO, G. O Fogo dos deuses: uma história da energia nuclear. Rio de Janeiro: Contraponto, 2006.

CENTRO DE DESENVOLVIMENTO DA TECNOLOGIA NUCLEAR. O CDTN. Brasília, DF: MCTI, [2016]. Disponível em: <http://www.cdtn.br/o-cdtn>. Acesso em: 5 set. 2016.

Pós-Graduação. Brasília, DF: MCTI, [2017]. Disponível em <https://goo.gl/fvTf4q>. Acesso em: 3 jan. 2017.

FILGUEIRAS, S. A. C. Indústria nuclear: estratégia tecnológica e parceria com um instituto de pesquisa. 2002. Tese (Doutorado em Engenharia) - Escola Politécnica da Universidade de São Paulo, São Paulo, 2002.

FIOLHAIS, C. Pré-História e história da Física Nuclear. Coimbra: Universidade de Coimbra, 2016. Disponível em: 〈https://goo.gl/5jxDx>. Acesso em: 12 dez. 2016.

INTERNATIONAL ATOMIC ENERGY AGENCY. Indicators for nuclear power development. Vienna: IAEA, 2015. Disponível em: <https://goo.gl/7TwuoD>. Acesso em: 2 dez. 2016.

KAMIOJI, M. I.; MAGALHÃES, G. S. Origins and evolution of the nuclear program in Brazil: the alliance between scientists and militaries for the institutionalization of Science \& Technology towards industrialization. In: WORLD ECONOMIC HISTORY CONGRESS, 17. 2015, Kyoto. Proceedings... Kyoto: Kyoto International Conference Center, 2015.

LA TRIBU CURIE. 26'26”. Abdelghani Boris. YouTube. 2014. Disponível em: $<$ https://goo.gl/YXYEzj>. Acesso em: 3 ago. 2016.

MARTINS, R. A. A maçã de Newton: história, lendas e tolices. In: SILVA, C. C. (Org.). Estudos de história e filosofia das ciências: subsídios para aplicação no ensino. São Paulo: Livraria da Física, São Paulo, 2006.

MESQUITA, A. Z. et al. The utilization of IPR-R1 TRIGA nuclear research reactor for educational purposes in Brazil. In: NUCLEAR EDUCATION AND TRAINING, 2011, Prague. Proceedings... Prague: European Nuclear Society, 2011. p. 295-299.

PEINADO, J.; RODACOSKI, G. C. A percepção da importância das disciplinas humanas e sociais na estrutura curricular por alunos de engenharia. Revista de Ensino de Engenharia, Brasília, DF, v. 27, n. 2, p. 20-33, 2008.

RÖHRLICH, E. Los átomos para la paz de Eisenhower: el discurso que inspiró la creación del OIEA. Boletín del OIEA, Vienna, v. 54, n. 4, p. 3-4, 2013. 
Mesquita e Moreira / Disciplina: história da Ciência - Tecnologia Nuclear, contribuição para a formação dos estudantes de pós-graduação em Engenharia

RONAN, C. A. História ilustrada da ciência da Universidade de Cambridge. São Paulo: Círculo do Livro, 1987.

ROSSONI, S. A história da ciência e do conhecimento: algumas (in)certezas. Revista de Ciências Humanas, Erechim, v. 4, n. 4, p. 1-22, 2003.

SANTOS, S. B. et al. A disciplina de história da ciência e da técnica: contribuições para o ensino e a formação de professores de química. Educación Química, Amsterdã, v. 25, n. 1, p. 71-81, 2014.

SCRIGNOLI, G. M. et al. As ciências humanas no curso de engenharia. In: SIMPÓSIO INTERNACIONAL DE INICIAÇÃO CIENTÍFICA E TECNOLÓGICA DA USP, 16., 2008, São Paulo. Anais... São Paulo: Escola Politécnica da Universidade de São Paulo, 2008.

SILVA FILHO, N. G.; SANTANA, J. G. L.; SILVA, L. R. B. A responsabilidade social na vida de um engenheiro. In: CONGRESSO BRASILEIRO DE EDUCAÇÃO EM ENGENHARIA, 39., 2011, Blumenau. Anais... Blumenau: Universidade Regional de Blumenau, 2011.

TENNENBAUM, J. Energia nuclear: uma tecnologia feminina. 2. ed. Rio de Janeiro: Capax Dei, 2007.

Recebido em 25/03/2017

Aprovado em 14/06/2017 